Published in final edited form as:

Gynecol Oncol. 2018 October ; 151(1): 1-3. doi:10.1016/j.ygyno.2018.09.005.

\title{
Tumor-infiltrating $\mathbf{T}$ cells in epithelial ovarian cancer: predictors of prognosis and biological basis of immunotherapy
}

\author{
Weimin Wang, \\ Department of Surgery, and University of Michigan Roger Cancer Center, University of Michigan \\ School of Medicine, Ann Arbor, Michigan, USA \\ Department of Obstetrics and Gynecology, and University of Michigan Roger Cancer Center, \\ University of Michigan School of Medicine, Ann Arbor, Michigan, USA \\ Weiping Zou, and \\ Department of Surgery, and University of Michigan Roger Cancer Center, University of Michigan \\ School of Medicine, Ann Arbor, Michigan, USA \\ Department of Pathology, and University of Michigan Roger Cancer Center, University of \\ Michigan School of Medicine, Ann Arbor, Michigan, USA \\ Center of Excellence for Cancer Immunology and Immunotherapy, and University of Michigan \\ Roger Cancer Center, University of Michigan School of Medicine, Ann Arbor, Michigan, USA

\section{J. Rebecca Liu} \\ Department of Surgery, and University of Michigan Roger Cancer Center, University of Michigan \\ School of Medicine, Ann Arbor, Michigan, USA \\ Department of Obstetrics and Gynecology, and University of Michigan Roger Cancer Center, \\ University of Michigan School of Medicine, Ann Arbor, Michigan, USA
}

\begin{abstract}
Epithelial ovarian cancer (EOC) is the most lethal gynecological malignancy worldwide, and is responsible for more than 100,000 deaths per year. EOC is a heterogeneous disease and includes with multiple histological subtypes [1]. High-grade serous ovarian cancer (HGSOC), the most common histologic subtype, accounts for more than $70 \%$ of EOC and portends the worst prognosis. The majority of HGSOC patients are diagnosed at advanced stage III or IV disease with metastasis throughout the abdomen and pelvis [2]. Surgical debulking and platinum-based chemotherapy have been the first-line therapy for patients with HGSOC for more than 30 years. Although initial response is often excellent, the disease usually recurs within 2-5 years as tumor cells develop resistance to chemotherapy [3]. Numerous favorable prognostic indicators for HGSOC have been reported, including early stage, optimal surgical debulking and sensitivity to platinum based chemotherapy [4].
\end{abstract}

Author Contributions

W.W.: conception, drafting, final approval of the version to be published; W.Z.: conception, editing, final approval of the version to be published; and J. R.L.: conception, revision, editing, final approval of the version to be published.

Conflicts of Interest

The authors have no conflicts of interest to report. 
Emerging evidence has shown that host immunity significantly influences cancer prognosis, and can be assessed by evaluating systemic inflammatory response and tumor-infiltrating immune cells [5,6]. Neutrophil-to-lymphocyte ratio (NLR) is known as a reliable measure of systemic inflammation and has predictive value in the early diagnosis of many types of cancer, including pancreatic, colorectal and ovarian cancers. Elevated levels of NLR have been associated with poor prognosis in cancer patients [7]. Serum lactate dehydrogenase (LDH) level has been shown to correlate with systemic inflammation and serves as a prognostic predictor of overall survival (OS) in several tumor types. Previous work suggests that serum LDH level can predict OS in patients with pancreatic cancer after chemotherapy [6]. In ovarian cancer patients, an earlier study suggested that serum LDH level in cancer patients was significantly higher than the LDH levels in patients with benign tumors [8]. However, the prognostic value of serum LDH in ovarian cancer has not been clarified.

Ovarian cancer is defined as an immunogenic tumor that exhibits a spontaneous antitumor immune response. Tumor-infiltrating lymphocytes (TILs) have long been known to exist in the ovarian tumor micro-environment and strongly correlate with favorable prognosis $[9,10]$. TILs are a type of white blood cells, and include T-cells, B-cells, macrophages or natural killer cells, localized in tumor islets and stroma. TILs are thought to play an important role in the control of tumor growth by activating anti-tumor immune response. Tumor cells are sampled by antigen presenting cells (APCs), and tumor-specific antigens are processed in APCs and presented to T-cells, which are then activated to $\mathrm{CD} 8^{+}$cytotoxic T-cells to directly recognize and eliminate tumor cells or $\mathrm{CD}^{+}$helper T-cells which regulate immune response through cytokine secretion and stimulation of other immune cells. Thus, in theory, tumorinfiltrating T-cells, in particular $\mathrm{CD}^{+}$and $\mathrm{CD} 8^{+} \mathrm{T}$ cells are strong prognostic indicators of clinical outcomes. Other investigators have shown that the presence of $\mathrm{CD}^{+} \mathrm{T}$-cells in EOC correlate with improved clinical outcomes [10]. Sato et al. demonstrated the prognostic significance of the ratio of $\mathrm{CD}^{+} \mathrm{T}$ cells versus FOXP3+ Treg cells in ovarian cancer [11]. Several subsequent studies confirmed that the presence of $\mathrm{CD}^{+} \mathrm{T}$ cells was associated with improved survival in ovarian cancer patients $[12,13]$. Although $\mathrm{CD}^{+} \mathrm{T}$ cells are usually non-cytotoxic, they can help recruit and activate $\mathrm{CD} 8^{+} \mathrm{T}$ cells to contribute to anti-tumor response. Several reports indicate that the presence of $\mathrm{CD} 4^{+}$TILs also correlate with improved outcomes in ovarian cancer patients [12].

In this issue, Mauricio et al. evaluated systemic inflammation status by measuring NLR and serum LDH level in a cohort of 128 HGSOC patients. Consistent with previous reports, they found that patients with increased NLR had significantly lower progression-free survival (PFS) and lower overall survival (OS) [14]. Moreover, in a multivariate analysis, HGSOC patients with higher serum levels of LDH displayed significantly lower OS but not PFS. These authors found a correlation between elevated NLR and increased LDH, suggesting that changes in these two parameters may be the result of increased systemic inflammation. Nevertheless, the results indicate that systemic inflammation is an early determinant of OS in HGSOC [14]. Although similar conclusions have been drawn by previous studies, this study confirms the notion that increased systemic inflammation correlates with poor survival in HGSOC in a cohort of Chilean patients. 
Next, to study the prognostic value of TILs, the authors quantified the amounts of $\mathrm{CD}^{+}$, $\mathrm{CD}^{+}$and $\mathrm{CD} 8^{+} \mathrm{T}$ cells in both tumor islets and stroma by immunohistochemistry staining. Intraepithelial CD3 correlated with better OS and PFS in both univariate and multivariate analyses. Both intraepithelial CD4 and CD8 were associated with better OS and PFS in univariate analysis; and the analysis using multivariate Cox modeling confirmed that intraepithelial CD4 was independently associated with better PFS and OS. Intraepithelial $\mathrm{CD}^{+}$was associated only with improved PFS but not OS. Overall, the authors concluded that tumor-infiltrating $\mathrm{CD} 4^{+}$and $\mathrm{CD} 8^{+} \mathrm{T}$ cells positivity correlate with patient survival in HGSOC [14]. In addition, programmed death-ligand 1 (PD-L1) expression was quantified and found to be associated with improved PFS and OS in the cohort, which was unexpected based on previous reports [14]. All in all, the study suggests that a combined score using systemic inflammation and TILs may be a better prognostic factor for HGSOC.

In all subtypes of TILs, $\mathrm{CD} 8^{+} \mathrm{T}$ cells are considered as a critical component with the most antitumor activity. $\mathrm{CD}^{+} \mathrm{T}$ cells execute tumor clearance by several mechanisms. First, they can recognize the specific antigens expressed on tumor surface, release cytotoxic molecular granzyme B and perforin, which are delivered into tumor cells and induce tumoral apoptosis. Second, $\mathrm{CD} 8^{+} \mathrm{T}$ cells can induce cancer cell death through the Fas/Fas ligand pathway. Third, cytokines released by $\mathrm{CD} 8^{+} \mathrm{T}$ cells, such as IFN $\gamma$ and TNF $a$, can have antitumor activities by inducing cancer cell senescence. Therefore tumor-infiltrating $\mathrm{CD} 8^{+} \mathrm{T}$ cells are associated with improved survival in almost all solid tumor types [15]. However, tumor progression can still occur despite the presence of tumor-reactive $\mathrm{CD} 8^{+} \mathrm{T}$ cells in the tumor microenvironment. We now know that cancer cells can create an immunosuppressive microenvironment by releasing inhibitory cytokines, expressing inhibitory molecules and recruiting immunosuppressive cells to down-regulate the antitumor activity of $\mathrm{CD} 8^{+} \mathrm{T}$ cells [16]. The PD-L1 and PD-1 pathway is one of the major inhibitory regulators mediating suppression of T cells. PD-L1 is widely expressed on tumor, stromal and immune cells. Its receptor PD- 1 is expressed on $\mathrm{T}$ cells, including $\mathrm{CD}^{+} \mathrm{T}$ cells, inhibits effector T-cell activity mainly in the effector phase in peripheral tissues and the tumor microenvironment [17]. PD-L1 and PD- 1 engagement can drive $C D 8^{+} \mathrm{T}$ cells into exhaustion. Exhausted CD8 ${ }^{+}$ $\mathrm{T}$ cells thus exhibit defects in proliferation and decreased cytokine production and cytotoxic functions. Antibodies targeting either PD-1 or PD-L1 result in abrogation of the inhibitory signal, thus restoring the function of $\mathrm{CD}^{+} \mathrm{T}$ cells and mediating tumor regression [17].

To date, the immunotherapy utilizing PD-L1 and PD-1 blockades have become the most promising treatment modality for numerous types of cancer, including melanoma, lung cancer, kidney cancer, bladder cancer, head and neck cancers, and Hodgkins lymphoma [18]. The application of PD blockade in EOC is still being tested in clinical trials. TILs, as the basis of effective PD blockade therapy, have also been recognized as a predictive biomarker for immunotherapy response. Mauricio et al.'s study confirms the correlation between CD8 ${ }^{+}$ T cells and improved outcome in patients with HGSOC, further highlighting the promise of immunotherapy in the treatment of ovarian cancer. Data from current studies also suggests that single-agent PD blockade is promising in EOC, but the overall response rate is much lower compared with clinical responses achieved in melanoma, lung cancer and renal cell cancer [19]. This suggests other immunosuppressive mechanisms may contribute to the resistance toward PD blockade in EOC. Combinatorial therapeutic strategies will be required 
to unleash the maximal antitumor immune response in the management of this disease [20]. Combining PD blockade with other biologic agents is now recognized as one of the most promising anti-neoplastic therapeutic strategies. For EOC, the combination of $\mathrm{PD}(\mathrm{L})-1$ antibodies with PARP inhibitors, VEGF inhibitors or conventional chemotherapy have achieved good response in clinical trials and demonstrate excellent clinical potential in ovarian cancer.

Another immunotherapy strategy is adoptive T-cell transfer, which consists of the infusion of ex vivo-expanded tumor-specific $\mathrm{T}$ cells generated from TILs or genetically modified $\mathrm{T}$ cells, such as chimeric antigen receptor (CAR) expressing T cells. ACT functions by directly increasing the number of tumor-reactive $\mathrm{CD}^{+}$effector $\mathrm{T}$ cells in tumor [21]. To date, two CAR T-cell therapies have been approved for the treatment of refractory diffuse large B-cell lymphoma (DLBCL) and refractory B-cell precursor acute lymphoblastic leukemia (ALL). ACT strategies have been also tested in preclinical ovarian cancer models, and are currently being investigated in early phase trials in EOC. Neoantigen-derived cancer vaccines, as another novel immunotherapy strategy, have recently been tested in patients with melanoma. These studies indicate that neoantigen vaccines can expand the repertoire of neoantigenspecific $\mathrm{CD}^{+}$and $\mathrm{CD} 8^{+} \mathrm{T}$ cells and mediate tumor regression [22,23]. Therefore, neoantigen vaccines represent another novel strategy of personalized immunotherapy for patients with EOC.

In conclusion, the infiltration of $\mathrm{T}$ cells into the tumor microenvironment is a favorable prognostic feature for EOC. A high density of intratumoral $\mathrm{CD}^{+}$or $\mathrm{CD} 8^{+} \mathrm{T}$ cells is associated with longer PFS and OS. Intratumoral $\mathrm{T}$ cells are also the basis of cancer immunotherapy. Current immunotherapy strategies, including checkpoint blockade, ACT and cancer vaccine, all converge on the tumor-specific $\mathrm{T}$ cells, especially $\mathrm{CD} 8^{+} \mathrm{T}$ cells.

\section{References}

[1]. Jayson GC, Kohn EC, Kitchener HC, Ledermann JA, Ovarian cancer, Lancet 384 (9951) (10 11 2014) 1376-1388. [PubMed: 24767708]

[2]. Bowtell DD, Bohm S, Ahmed AA, et al. Rethinking ovarian cancer II: reducing mortality from high-grade serous ovarian cancer, Nat. Rev. Cancer 15 (11) (11 2015) 668-679. [PubMed: 26493647]

[3]. Wang W, Kryczek I, Dostal L, et al. Effector T Cells Abrogate Stroma-Mediated Chemoresistance in Ovarian Cancer, Cell 165 (5) (5 19 2016) 1092-1105. [PubMed: 27133165]

[4]. Au KK, Josahkian JA, Francis JA, Squire JA, Koti M, Current state of biomarkers in ovarian cancer prognosis, Future Oncol 11 (23) (2015) 3187-3195. [PubMed: 26551891]

[5]. Zhang H, Lu J, Lu Y, et al. Prognostic significance and predictors of the system in-flammation score in ovarian clear cell carcinoma, PLoS One 12 (5) (2017), e0177520.. [PubMed: 28498842]

[6]. Yu SL, Xu LT, Qi Q, et al. Serum lactate dehydrogenase predicts prognosis and correlates with systemic inflammatory response in patients with advanced pancreatic cancer after gemcitabinebased chemotherapy, Sci. Rep 7 (3 27 2017) 45194. [PubMed: 28345594]

[7]. Templeton AJ, McNamara MG, Seruga B, et al. Prognostic role of neutrophil-tolymphocyte ratio in solid tumors: a systematic review and meta-analysis, J. Natl. Cancer Inst 106 (6) (6 2014), dju124. [PubMed: 24875653]

[8]. Boran N, Kayikcioglu F, Yalvac S, Tulunay G, Ekinci U, Kose MF, Significance of serum and peritoneal fluid lactate dehydrogenase levels in ovarian cancer, Gynecol. Obstet. Investig 49 (4) (2000) 272-274. [PubMed: 10828712] 
[9]. Santoiemma PP, Powell DJ, Jr., Tumor infiltrating lymphocytes in ovarian cancer, Cancer Biol. Ther 16 (6) (2015) 807-820. [PubMed: 25894333]

[10]. Zhang L, Conejo-Garcia JR, Katsaros D, et al. Intratumoral T cells, recurrence, and survival in epithelial ovarian cancer, N. Engl. J. Med 348 (3) (1 16 2003) 203-213. [PubMed: 12529460]

[11]. Sato E, Olson SH, Ahn J, et al. Intraepithelial CD8+ tumor-infiltrating lymphocytes and a high $\mathrm{CD} 8+$ /regulatory $\mathrm{T}$ cell ratio are associated with favorable prognosis in ovarian cancer, Proc. Natl. Acad. Sci. U. S. A 102 (51) (12 20 2005) 18538-18543. [PubMed: 16344461]

[12]. Li J, Wang J, Chen R, Bai Y, Lu X, The prognostic value of tumor-infiltrating T lymphocytes in ovarian cancer, Oncotarget 8 (9) (2 28 2017) 15621-15631. [PubMed: 28152503]

[13]. Nielsen JS, Sahota RA, Milne K, et al. CD20+ tumor-infiltrating lymphocytes have an atypical $\mathrm{CD} 27-$ memory phenotype and together with $\mathrm{CD} 8^{+} \mathrm{T}$ cells promote favorable prognosis in ovarian cancer, Clin. Cancer Res 18 (12) (6 15 2012) 3281-3292. [PubMed: 22553348]

[14]. Pinto MP, Balmaceda C, Bravo ML, et al. Patient inflammatory status and CD $4^{+} / \mathrm{CD}^{+}$ intraepithelial tumor lymphocyte infiltration are predictors of outcomes in high-grade serous ovarian cancer, Gynecol. Oncol 151 (1) (2018) 10-17. [PubMed: 30078505]

[15]. Wang W, Green M, Rebecca Liu J, Lawrence TS, Zou W, CD8+ T Cells in Immuno-therapy, Radiotherapy, and Chemotherapy, in: Zitvogel L, Kroemer G (Eds.), Oncoimmunology: A Practical Guide for Cancer Immunotherapy, Springer International Publishing, Cham 2018, pp. 23-39.

[16]. Zou W, Immunosuppressive networks in the tumour environment and their therapeutic relevance, Nat. Rev. Cancer 5 (4) (4 2005) 263-274. [PubMed: 15776005]

[17]. Zou W, Wolchok JD, Chen L, PD-L1 (B7-H1) and PD-1 Pathway Blockade for Cancer Therapy: Mechanisms, Response Biomarkers and Combinations, Sci. Transl. Med 8 (2016), 328rv324.

[18]. Topalian SL, Drake CG, Pardoll DM, Immune checkpoint blockade: a common denominator approach to cancer therapy, Cancer Cell 27 (4) (4 13 2015) 450-461. [PubMed: 25858804]

[19]. Weiss L, Huemer F, Mlineritsch B, Greil R, Immune checkpoint blockade in ovarian cancer, Memo 9 (2016) 82-84. [PubMed: 27429660]

[20]. Swart M, Verbrugge I, Beltman JB, Combination Approaches with Immune-Checkpoint Blockade in Cancer Therapy, Front. Oncol 6 (2016) 233. [PubMed: 27847783]

[21]. Restifo NP, Dudley ME, Rosenberg SA, Adoptive immunotherapy for cancer: harnessing the T cell response, Nat. Rev. Immunol 12 (4) (3 22 2012) 269-281. [PubMed: 22437939]

[22]. Sahin U, Derhovanessian E, Miller M, et al. Personalized RNA mutanome vaccines mobilize poly-specific therapeutic immunity against cancer, Nature 547 (7662) (7 13 2017) 222-226. [PubMed: 28678784]

[23]. Ott PA, Hu Z, Keskin DB, et al. An immunogenic personal neoantigen vaccine for patients with melanoma, Nature 547 (7662) (7 13 2017) 217-221. [PubMed: 28678778]

Gynecol Oncol. Author manuscript; available in PMC 2019 October 01. 